\title{
A Planning Approach of Engineering Characteristics Based on QFD-TRIZ Integrated
}

\author{
Shang $\mathrm{Liu}^{1}$, Dongyan $\mathrm{Shi}^{1,2}$, and Ying Zhang ${ }^{1}$ \\ ${ }^{1}$ College Mechanical and Electrical Engineering, Harbin Engineering University, \\ Harbin 150001, China \\ ${ }^{2}$ Postdoctoral Research Station of Instrument Science and Technology, Harbin University of \\ Science and Technology, Harbin 150001, China \\ \{Shang.Liu, Dongyan Shi, Ying. Zhang, QQLSCN\}@126.com
}

\begin{abstract}
Traditional QFD planning method compromises contradictions between engineering characteristics to achieve higher customer satisfaction. However, this compromise trade-off can not eliminate the contradictions existing among the engineering characteristics which limited the overall customer satisfaction. QFD (Quality function deployment) integrated with TRIZ (the Russian acronym of the Theory of Inventive Problem Solving) becomes hot research recently for TRIZ can be used to solve contradictions between engineering characteristics which construct the roof of HOQ (House of quality). But, the traditional QFD planning approach is not suitable for QFD integrated with TRIZ for that TRIZ requires emphasizing the contradictions between engineering characteristics at problem definition stage instead of compromising trade-off. So, a new planning approach based on QFD / TRIZ integration is proposed in this paper, which based on the consideration of the correlation matrix of engineering characteristics and customer satisfaction on the basis of cost. The proposed approach suggests that TRIZ should be applied to solve contradictions at the first step, and the correlation matrix of engineering characteristics should be amended at the second step, and at next step IFR (ideal final result) must be validated, then planning execute. An example is used to illustrate the proposed approach. The application indicated that higher customer satisfaction can be met and the contradictions between the characteristic parameters are eliminated.
\end{abstract}

Keywords: QFD, TRIZ, product planning, engineering characteristics.

\section{Introduction}

Product planning is the key step at the early stage of products design. Quality function deployment (QFD) became a well known customers-oriented product development tool for translating 'customer voice' into various stages of product design including product planning stage. As the important parameters of product planning, engineering characteristics (ECs) constructing the roof of house of quality (HoQ) incarnate the customers' requirements (CRs). So a reasonable product planning is the base of meeting overall customer satisfaction. In the last decade, product planning based on QFD has been undertaken by researcher [1-5]. But all these approach mentioned above have the same means to deal with contradictions between ECs, which is compromise trade-off. 
QFD can solve the problem of "what to do", but can't answer the question of "how to do", and TRIZ provides a series of problem-solving tools, so integrated QFD and TRIZ can solve the problems of "what to do " and "how to do" in design process [6]. The methods of QFD integrated with TRIZ were discussed in papers [7-9].

TRIZ (Theory of Inventive Problem Solving) considers that technology system evolves towards IFR (ideal final result), and the resolve of contradictions between design parameters is the driving forces of technology systems evolution. TRIZ promotes emphasizing the contradictions between parameters at problem definition stage instead of compromising trade-off. Therefore, the product planning method based on the traditional QFD is not suitable for QFD / TRIZ integrated design model, a new planning approach is needed. This paper proposes a design method of product planning based on QFD and TRIZ contradictions tools integrated, as well as the consideration to the analysis of the correlation matrix of ECs and the constraints of exploitation cost.

\section{QFD/TRIZ Integerated Product Planning Methods}

\subsection{Planning Process Model}

The planning process of proposed approach is shown in Figure 1, which suggest a five step planning process. The first step is to construct the HOQ. Correlation matrix which composed of the roof of HOQ is the key to QFD integrated with TRIZ for that it is not only important for identifying the weight of ECs but also important for

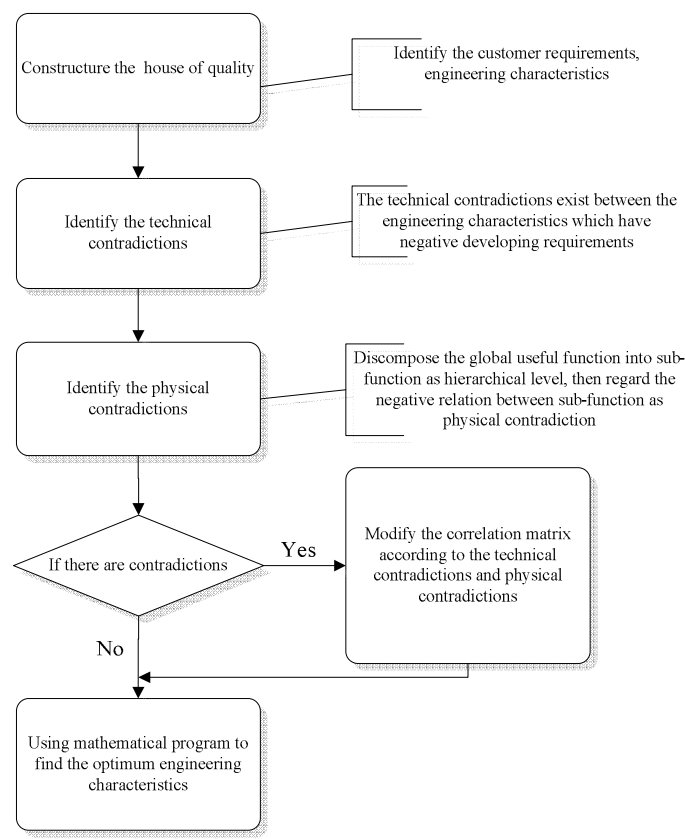

Fig. 1. Engineering characteristics planning process model 
applying TRIZ. The second step is to identify the technical contradictions. The technical contradictions represent the interactive constraints of ECs, which limit the overall customer satisfaction. If the contradiction can be eliminated at the early stage of product planning, higher customer satisfaction will be met. The details will be illustrated in section 2.3. The third step is to identify the physical contradictions existing in the correlation matrix, and the eliminating operation will also be executed to meet higher overall customer satisfaction, and the details will be illustrated in section 2.4. The forth step is verification. The style of contradiction should be distinguished. If the contradiction is technical contradiction, contradiction matrix will be employed to solve the problem. If the contradiction is physical contradiction, separating principle will be applied. Then the IFR validation in step 4 is essential for cost consideration. In the last step, the nonlinear programming based on Chen Yizeng's approach is employed to deal with optimizing problem.

\subsection{Constructing HoQ}

Constructing HoQ involves the collection of expert opinion to identify the data shown as follow:

1. Customer requirements which construct the left wall of HoQ are denoted by $C R \mathrm{~s}$, and $C R_{\mathrm{i}}$ represent the i-th customer requirement.

2. Engineering characteristics which construct the ceiling of HoQ is denoted by $E C s$, and $E C_{j}$ represent the j-th engineering characteristic.

3. Correlation matrix which constructs the roof of $\mathrm{HoQ}$ is denoted by $P$, and represents the interactive relationship of engineering characteristics. $p_{i j}$ is the element of $P$, represent the interactive strength between $E C_{i}$ and $E C_{j}$, where $p_{i j} \in(-1,1]$.

4. Relation matrix which reflect the relationship between $C R s$ and $E C s$ construct the room of HoQ, and is denoted by $R . r_{i j}$ is the element of $R$ represent interactive strength between $C R_{i}$ and $E C_{j}$.

5. The engineering characteristics' value from competitive company which constructs the floor of HoQ is used to be reference.

6. The upper limit denoted by $l_{\max }$ and lower limit denoted by $l_{\min }$ of $E C s$ is essential for product planning which are set by the developer according to the physical limit and the parameters of competitive enterprise.

7. Cost coefficient is essential for product planning according to Chen Yizeng's approach, which constructs the foundation of HoQ, denoted by $c f_{j}^{*}$.

\subsection{Identifying Technical Contradictions}

The interactive relationship between engineering characteristics can be classified into positive constraint relationship and negative constraint relationship. The positive 
constraint relationship means if the i-th engineering characteristic should be improved, the $\mathrm{j}$-th engineering characteristic need to be improved at the same time. The negative constraint relationship mean if the i-th engineering characteristic should be improved, the $\mathrm{j}$-th engineering characteristic need to be worsened at the same time. And the negative constraint relationship which is called contradiction in TRIZ can be mapped to contradiction matrix to find the solution. A method is come up with in reference [10] to find the technical contradiction from HOQ. As is shown in Fig.2, there is a contradiction between $E C_{i}$ and $E C_{j}$. Then $E C_{i}$ is mapped to $P_{m}$ (TRIZ engineering parameter for improving parameter) and $E C_{j}$ is mapped to $P_{n}$ (TRIZ engineering parameter for worsening parameter). The solutions exist at the cross of row $m$ and column $n$. This solution is applied to improve the capability of system and the contradiction between $E C_{i}$ and $E C_{j}$ will be eliminated.

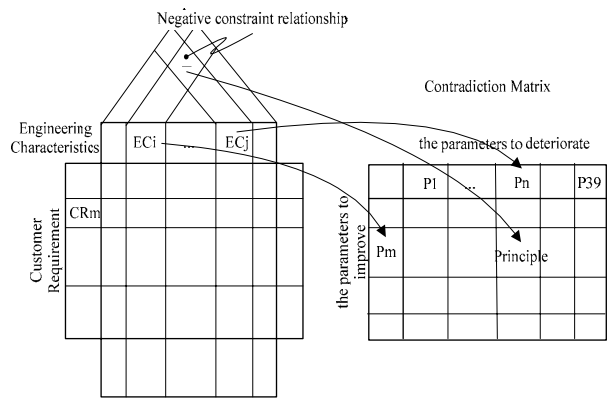

Fig. 2. Model of HoQ integrated with contradiction matrix

\subsection{Identifying Physical Contradictions}

Engineering characteristics need to grow in both positive and negative direction at the same time for the requirement of improving capability of product, which is called physical contradiction in TRIZ. An approach combined QFD with function decomposition to solve the physical contradictions was proposed in reference [11]. As is shown in Fig.3, system function can be decomposed into several sub-function from 1 to $n$ which are denoted by $S F_{1}, S F_{2} \cdots S F_{n}$. And these functions are affected by engineering characteristics. For example, a positive change requirement on $E C_{j}$ emerge to improve sub-function $S F_{x}$, meanwhile a negative change requirement on $E C_{j}$ emerge to improve sub-function $S F y$, so the physical contradiction emerge on engineering characteristic $E C_{j}$. Separating principle is proposed to eliminate the physical contradiction. 


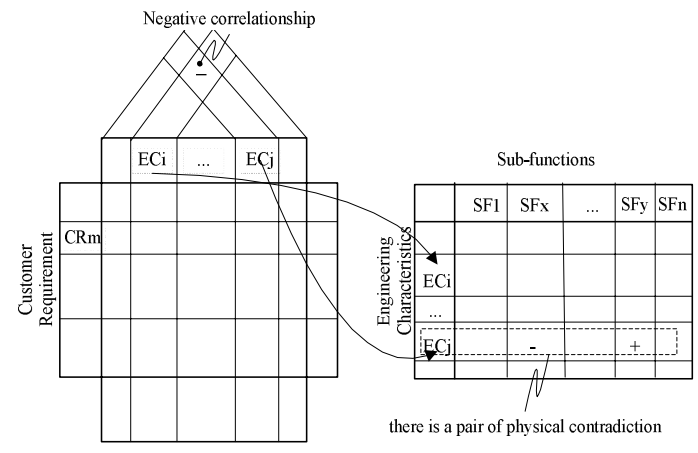

Fig. 3. Mapping of HoQ integrated with decomposed function

\subsection{Dealing with Correlation Matrix of Engineering Characteristics}

Contradictions and constraints exist in engineering characteristics, which influence the improvement of engineering characteristics. Positive correlation is known as constraint and negative correlation is known as contradiction. In customer satisfaction planning process based on the consideration of cost, the existence of constraints can't limit customer satisfaction, but the corresponding improve of engineering characteristics will be limited by development cost. Customer satisfaction will be limited for the existence of contradictions. Customer satisfaction will improve if these contradictions can be eliminated. TRIZ is employed to eliminate these contradictions. Therefore, the matrix of engineering characteristics is done as follows:

1. Keep constraint relations to constraint items.

2. Original contradiction relationships will be saved if solutions can't be found to solve the contradictions by TRIZ to the contradictions items. If solutions can be found by using TRIZ, then IFR must be validated. If the validation is successful, these contradictions will be revised to 0 , otherwise the original relationship of contradiction will be kept.

TRIZ IFR validating method:

TRIZ theory considers that resolve of contradictions between design parameters is the driving force of technology systems evolution. Technology system evolves towards improvement of IFR and IFR can be expressed as $I=\frac{\sum U F}{\sum H F+\sum C}$. Where $I$ is the ideal degree of technology systems, and $U F$ is useful function of systems, and $H F$ is harmful function of system, and $C$ is the cost. Obviously, in order to improve the ideal degree of technology systems, it is necessary to increase the useful functions, and reduce harmful function, or reduce the cost. In application of TRIZ to solve the contradictions, system resources should be considered. Whether the 
solutions generated by TRIZ will give rise to extra cost must be considered. If it won't cause extra cost, the validation is successful.

\subsection{Nonlinear Programming}

Based on Chen Yizeng's approach [1], we come up with a systematic flow in product planning. It is composed of nine stages.

1. To identify the weight importance of customer requirements which is denoted by Vector $W . w_{i}$ represents the $\mathrm{i}$-th element of $W$ corresponding to weight importance of the i-th customer requirement. And analytical hierarchy process (AHP)[12] is employed to calculate the value of $W$, where $0<w_{i}<1, \quad \sum_{i=1}^{m} w_{i}=1$, and $m$ is the count of customer requirements.

2. To identify the actual improved ratio [1] and the plan improved ratio [1] of engineering characteristics. The plan improved ratio denoted by $x$ is defined as the percent of engineering characteristic which should be reaching in order to improve the capability of product without considering other engineering characteristics' effect. The actual improved ratio denoted by $y$ is defined as the percent of engineering characteristic which should be reaching in order to improve the capability of product with considering other engineering characteristics' effect. The $\mathrm{j}$-th engineering characteristic's actual improved ratio $y_{j}$ can be calculated by expressions $y_{j}=\left(x_{j}+\sum_{\substack{k=1 \\ k \neq j}}^{n} x_{k} p_{j k}\right)$. Where $x_{j}$ is the jth engineering characteristic's plan improved ratio and $p_{j k}$ is the element of correlation matrix $P$ represent the relation strength between $E C_{i}$ and $E C_{j}$. The count of engineering characteristics is denoted by $n$.

3. To identify the customer satisfaction which is denoted by Vector $U$ and $u_{i}$ is the ith element of Vector $U$ corresponding to the ith customer satisfaction, which can be calculated by expressions $U=R X$. Where $X$ represents the plan improved ratio Vector.

4. To identify the weight importance of $E C_{j}$ which is denoted by Vector $V$, where $V=R^{T} W$. Then normalize the Vector $V$, we got $V^{*}=\left[v_{1}^{*}, v_{2}^{*}, v_{3}^{*}, \ldots . . v_{n}^{*}\right]^{T}, v_{j}^{*}=\frac{v_{j}}{\sum_{j=1}^{n} v_{j}}, 0<v_{j}^{*}<1, \sum_{j=1}^{n} v_{j}^{*}=1$. 
5. To identify the overall customer satisfaction which is denoted by function $S(X)$.

$$
S(X)=\left(V^{*}\right)^{T} X=\sum_{k=1}^{n} v_{k}^{*} x_{k}
$$

6. To create cost constraint functions according to the expressions $C\left(y_{j}\right)=c f_{j} y_{j}$, where $c f_{j}$ is cost coefficient represent the cost for improving the $\mathrm{j}$-th engineering characteristic and can be calculates by expressions $c f_{j}=c f_{j}^{*} \times\left(1-\sum_{\substack{k=1 \\ k \neq j}}^{n} x_{k} p_{j k}\right)$. $c f_{j}^{*}$ is the plan cost coefficient of $E C_{j}$.

7. Creating other constraint functions according to boundary condition.

8. Modifying the correlation matrix $P$ according to the result of applying of TRIZ.

9. Simulation.

10.Then, the nonlinear programming model is shown as follow:

$$
\begin{array}{ll}
\max & S(X)=\left(V^{*}\right)^{T} X=\sum_{k=1}^{n} v_{k}^{*} x_{k} \\
\text { s.t. } & \sum_{j=1}^{n} c f_{j}^{*}\left(1-\sum_{\substack{k=1 \\
k \neq j}}^{n} p_{j k} x_{j}\right)\left(x_{j}+\sum_{\substack{k=1 \\
k \neq j}}^{n} p_{j k} x_{j}\right) \leq T \\
& \alpha_{j} \leq \sum_{k=1}^{n} p_{j k} x_{j} \leq 1 \\
& 0 \leq x_{k} \leq 1 \\
& (j=1,2, \cdots, n)
\end{array}
$$

\section{Illustrated Example and Simulation Results}

Verify the reasonableness of the proposed approach in this paper by using examples of reference [1].

There are four customer demands $\left(C R_{1}, C R_{2}, C R_{3}, C R_{4}\right)$, seven engineering characteristic parameters $\left(E C_{1}, E C_{2}, \cdots, E C_{7}\right)$, and two competition enterprises $\left(\operatorname{comp}_{1}, \operatorname{comp}_{2}\right)$. Product house of quality is shown in Fig. 4.

Relative weight of customer requirements $W$ can be calculated by the method of AHP, and $W=(0.46,0.28,0.16,0.1)$. Correlation matrix of customer requirements and engineering characteristics denoted by $R$ can be identified from the room of 


\begin{tabular}{|c|c|c|c|c|c|c|c|c|c|c|c|c|}
\hline \multirow{2}{*}{\multicolumn{5}{|c|}{$\star$ positive strong correlation }} & \begin{tabular}{|c|} 
Engineering \\
Characteristics
\end{tabular} & $E C_{1}$ & $E C_{2}$ & $\mathrm{EC}_{3}^{+}$ & $E C_{+}$ & $E C_{9}^{+}$ & $E C \cdot$ & $E C_{7}$ \\
\hline & & & & & $E C_{1}$ & $\star$ & 2 & 2 & 3 & + & 5 & 3 \\
\hline \multirow{2}{*}{\multicolumn{5}{|c|}{ positive moderate correlation }} & $E C_{2}$ & s & $\star$ & 2 & e & 4 & 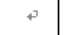 & 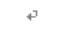 \\
\hline & & & & & $E C_{3}$ & $P$ & 8 & $\star$ & 8 & 2 & 8 & 3 \\
\hline \multirow{4}{*}{\multicolumn{5}{|c|}{$\begin{array}{l}\Delta \text { positive weak correlation } \\
\Delta \text { negative weak correlation }\end{array}$}} & $E C_{4}$ & 4 & $\mathbf{0}$ & 3 & $\star$ & 2 & $\Delta$ & 8 \\
\hline & & & & & $E C$ & 4 & 2 & 4 & 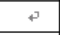 & $\star$ & 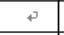 & 4 \\
\hline & & & & & $E C_{6}$ & 2 & 2 & 2 & $\Delta$ & 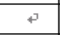 & $\star$ & 2 \\
\hline & & & & & $E C_{7}$ & 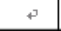 & 3 & 3 & 4 & 4 & 8 & $\star$ \\
\hline \begin{tabular}{l|} 
Customer \\
Requirement
\end{tabular} & $C R_{1}+$ & $C R_{2}+$ & $C R_{3}+$ & $C R_{+}+$ & $\begin{array}{l}\text { Relative } \\
\text { Weights }\end{array}$ & 0.13 & 0.30 & 0.17 & 0.24 & 0.08 & 0.03 & 0.05 \\
\hline$C R_{1}$ & 1 & 2 & 3 & 4 & 0.46 & $\boldsymbol{\theta}$ & $\star$ & $\boldsymbol{\theta}$ & 4 & $P$ & 2 & 4 \\
\hline$C R_{2}$ & 0.5 & 1 & 2 & 3 & 0.28 & 8 & 2 & $\boldsymbol{\Lambda}$ & $\star$ & $\mathbf{a}$ & 2 & 3 \\
\hline $\mathrm{CR}_{3}$ & 0.33 & 0.5 & 12 & 2 & 0.16 & 8 & 5 & 4 & 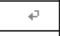 & 4 & $\star$ & 2 \\
\hline$C R_{4}$ & 0.25 & 0.33 & 0.5 & 1 & 0.10 & $?$ & 3 & 2 & 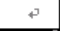 & 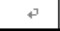 & 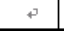 & $\star$ \\
\hline \multirow{6}{*}{\multicolumn{3}{|c|}{ 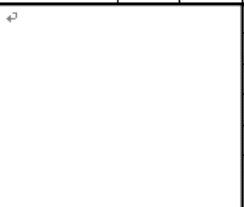 }} & \multicolumn{3}{|c|}{ Measurement units } & $m m^{-2+}$ & $\mathrm{mm}^{-2}+$ & $\mathrm{H}$ & $\mu_{m^{-2}}$ & HRC & $\mathrm{dB}$ & $m$ \\
\hline & & & \multicolumn{3}{|c|}{ Comp $p_{2}$} & 12 & 10 & 600 & 75 & 45 & 70 & 1. 75 \\
\hline & & & \multicolumn{3}{|c|}{ Comp $p_{2}$} & 8 & 5.6 & 40 & 85 & 50 & 70 & 1.8 \\
\hline & & & \multicolumn{3}{|c|}{ Minimum value } & 6 & 4 & 550 & 60 & 40 & 50 & 1.5 \\
\hline & & & \multicolumn{3}{|c|}{ Maximum value } & 15 & 12 & 700 & 100 & 60 & 90 & 2 \\
\hline & & & \multicolumn{3}{|c|}{$\operatorname{Cost}\left(c f_{j}^{*}\right)$} & 20 & 25 & 10 & 15 & 5 & 30 & 8 \\
\hline
\end{tabular}

Fig. 4. Product House of Quality

HoQ. Correlation matrix of engineering characteristics denoted by $P$ can be identify from the roof of HoQ, where

$$
P=\left[\begin{array}{ccccccc}
1 & 0 & 0 & 0 & 0 & 0 & 0 \\
0 & 1 & 0 & 0.56 & 0 & 0 & 0 \\
0 & 0 & 1 & 0 & 0 & 0 & 0 \\
0 & 0.56 & 0 & 1 & 0 & -0.33 & 0 \\
0 & 0 & 0 & 0 & 1 & 0 & 0 \\
0 & 0 & 0 & -0.33 & 0 & 1 & 0 \\
0 & 0 & 0 & 0 & 0 & 0 & 1
\end{array}\right]
$$

It can be seen from the matrix that there are technology contradictions between $E C_{4}$ and $E C_{6} . E C_{4}$ is loading efficiency, and $E C_{6}$ is cam driving noise. In order to improve the efficiency of loading efficiency, the speed of cam must be enhanced, but meanwhile it will increase driving noise (The reason is high-speed driving impact by the cam). Engineering characteristics are mapped to engineering parameters of TRIZ, $E C_{4}$ mapped to speed, and $E C_{6}$ mapped to Tension / Pressure, TRIZ principle numbers are required $(6,18,38$, and 40$)$, and the 40th principle is the composite materials. Stanyl PA46 material is adopted, then the cost less than the quondam cam and the noise is lower. The problems are solved, and the contradictions are removed, as well as not lead to additional cost. The contradictions are not found in engineering characteristics. 
Therefore, revise matrix $P$,

$$
P=\left[\begin{array}{ccccccc}
1 & 0 & 0 & 0 & 0 & 0 & 0 \\
0 & 1 & 0 & 0.56 & 0 & 0 & 0 \\
0 & 0 & 1 & 0 & 0 & 0 & 0 \\
0 & 0.56 & 0 & 1 & 0 & 0 & 0 \\
0 & 0 & 0 & 0 & 1 & 0 & 0 \\
0 & 0 & 0 & 0 & 0 & 1 & 0 \\
0 & 0 & 0 & 0 & 0 & 0 & 1
\end{array}\right]
$$

Set up mathematical programming model and constraint conditions according to expressions (1):

$\max S(x)=0.13 x_{1}+0.30 x_{2}+0.17 x_{3}+0.24 x_{4}+0.08 x_{5}+0.03 x_{6}+0.05 x_{7}$ s.t. $\quad 20 x_{1}+33.4 x_{2}+10 x_{3}+29 x_{4}+5 x_{5}+30 x_{6}+8 x_{7}-22.4 x_{2} x_{4}-4.7 x_{2}^{2}-$ $7.84 x_{4}^{2} \leq 80$

$$
\begin{aligned}
& 0.5 \leq x_{2}+0.56 x_{4} \leq 1 \\
& 0.5 \leq 0.56 x_{2}+x_{4} \leq 1 \\
& 0.5 \leq x_{1,3,5,6,7} \leq 1 \\
& 0 \leq x_{2,4} \leq 1
\end{aligned}
$$

Using MatLab optimization toolbox to solve, and optimize results are shown in Table 1.

Table 1. Simulation Result

\begin{tabular}{lccccccc}
\hline $\max S(X)$ & $x_{1}$ & $x_{2}$ & $x_{3}$ & $x_{4}$ & $x_{5}$ & $x_{6}$ & $x_{7}$ \\
\hline 0.7875 & 1.0000 & 0.6410 & 1.0000 & 0.6410 & 1.0000 & 0.3786 & 1.0000 \\
\hline
\end{tabular}

The results verify that overall customer satisfaction improves $5.75 \%$ compared with reference [1]'s.

\section{Conclusions}

To emphasizing the contradictions among the engineering characteristics instead of compromise trade-off at the early stage of product development is propitious to solve problem and promote the evolution of technology systems according to TRIZ. So traditional method for product planning based on the compromise trade-off is not suitable for the application in QFD integrated with TRIZ. A new product planning 
approach is proposed in this paper, we suggest that contradiction matrix and separating principle should be employed to eliminate the contradictions existing among the engineering characteristics before product planning, and IFR validate is essential for the cost consideration. Simulation result shows that the proposed approach can meet higher customer satisfaction, while the contradictions which baffle the development of technical system are eliminated.

\section{References}

1. Chen, Y., Tang, J., Ren, L., et al.: An Optimal Model Based on House of Quality. China Mechanical Engineering, 14967-14970 (2003)

2. Moskowitz, H., Kim, K.J.: QFD Optimizer: A Novice Friendly Quality Function Deployment Decision Support System For Optimizing Product Designs. Computers \& Industrial Engineering, 32641-32655 (1997)

3. Tang, J., Richard, Y.K.F., Xu, B., et al.: A new approach to quality function deployment planning with financial consideration. Computers \& Operations Research 29, 1447-1463 (2002)

4. Ertugrul Karsak, E., Sozer, S., Emre Alptekin, S.: Product planning in quality function deployment using a combined analytic network process and goal programming approach. Computers \& Industrial Engineering, 44171-44190 (2003)

5. Chen, L.-H., Ko, W.-C.: Fuzzy approaches to quality function deployment for new product design. Fuzzy Sets and Systems. In press, Corrected Proof, Available online December 24 (2008)

6. Tan, R.-H., Ma, J.-H., Zhang, H.-G., et al.: Study on the conceptual design process based on QFD and TRIZ. Machine Design 9 (2002)

7. Lu, C., Liao, Z., Jiang, S., et al.: Research on innovative product design system based on QFD and TRIZ. Materials Science Forum. In: Advances in Materials Manufacturing Science and Technology II - Selected Papers from the 12th International Manufacturing Conference in China, vol. 532-533, pp. 1144-1147 (2006)

8. Wang, H., Chen, G., Lin, Z., et al.: Algorithm of integrating QFD and TRIZ for the innovative design process. International Journal of Computer Applications in Technology, 2341-2352 (2005)

9. Shi, G.-1., She, Y.-g.: Study on problem-solving theory based on QFD, TRIZ and Taguchi methods. Systems Engineering and Electronics, 30851-30857 (2008)

10. Noel, L.: A new model of the conceptual design process using AFD/FA/TRIZ. TRIZ Journal, http: / /www.triz-journal . com

11. Armacost, R.L., Componation, P.J., Michael, et al.: An AHP Framework for prioritizing Customer Requirements in QFD. An Idustrialized Housing Application. IIE Transaction, 2672-2679 (1994)

12. $\mathrm{Mu}, \mathrm{R} .$, Zhang, J.-t.: Application of quality house technology in the refrigerator design based on qualitative function disposition. Journal of Machine Design, 2445-2448 (2007) 